\title{
Enseignements tirés de diagnostics d'inondabilité sur sinistres
}

\section{Lessons from flood sensitivity diagnostics after desasters}

\author{
par Y.P. Vuillaume
}

Hydro-Expert à Toulouse

The methodology used for auditing flooded areas in South western France, for insurance or legal purposes, consists in examining the spatial coherency and occurrence between rainfall - discharge - flood submersion, in order to define an hydraulic scheme of the site after a critical event. We use for that any available data, generally scarce.

On the basis of typical disasters, from single submersion to torrential flood, we observe the impact of urbanisation \& rural planning for sites generally sensitives.

So, for such exposed areas either local communities or industrial plants, and for insurance or security purposes, a preventive hydro audit is recommanded.

\section{I $\square$ INTRODUCTION}

Les nouvelles réglementations : la loi sur l'indemnisation des catastrophes naturelles, sur l'eau de 1992, celle de 1995 et l'implication éventuelle des personnes morales en matière pénale, combinées à une demande sociale en matière de recours, ont créé un nouveau contexte en ce qui concerne la prévention des inondations. La situation a donc évolué comme suit :

\section{Antérieurement}

Actuellement décennale $=$ indemnisation

\begin{tabular}{|c|c|c|}
\hline Occurrence & vingtennale & centennale $=$ PPR \\
\hline Indemnisation & circonstancielle & $\begin{array}{l}\text { automatique sur } \\
\text { arrêté préfectoral }\end{array}$ \\
\hline Financement & public, a posteriori & $\begin{array}{l}\text { anticipé : } 9 \% \rightarrow 12 \% \\
\text { assurance habitation }\end{array}$ \\
\hline Règle territoriale & traditionnelle & $\begin{array}{l}\text { inscription au POS, } \\
\text { opposable }\end{array}$ \\
\hline
\end{tabular}

Dans ce contexte le développement éventuel du sinistre en litige prend un nouveau relief.

\section{II —MODALITÉS D'UNE EXPERTISE D'INONDABILITÉ}

\subsection{Etapes d'une expertise sur sinistre}

Pour cela l'expert judiciaire intervient à titre individuel sur la base d'une mission spécifique, souvent à titre conservatoire pour la protection des intérêts du demandeur, ou éventuellement pour cause d'urgence. Par son indépendance des
Parties et la nature de sa mission, il apporte un point de vue global sur les facettes du sinistre : hydrologique, d'aménagement, réglementaire, social.

La démarche de l'expert est placée sous la contrainte du contradictoire entre les parties donc sous le contrôle de leurs avocats et éventuellement de leurs experts.

Cette expertise réunissant l'ensemble des Parties comprendra :

Les constatations in situ, avec confrontation des témoignages, examen des rapports éventuels des services administratifs \& audition de sachants éventuels ;

Les contrôles hydrologiques, parcellaire, d'aménagements, avec intervention selon le besoin d'un sapiteur (expert dans une autre discipline);

L'élaboration d'un rapport de diagnostic hydrologique sous forme d'un audit selon les règles de l'art, qu'il explicite aux Parties afin de recueillir leurs commentaires: les dires auxquels l'expert doit fournir une réponse écrite et techniquement argumentée ;

La définition des coûts de corrections ou de réparations ;

La définition des éléments du préjudice éventuellement subi par le Demandeur.

\subsection{Méthodologie de l'investigation expertale}

Elle procède d'un audit (et non pas d'une étude) fondé sur l'analyse technique (où les chroniques de données hydrologiques font souvent défaut), de la cohérence des relations pluie-débit-submersion dans sa distribution spatiale, établissement de schéma hydraulique localisé, et interaction avec les contraintes d'aménagement et du POS.

Elle répond aux questions posées par les parties et leurs conseils juridiques. 


\section{EXEMPLES DE DIAGNOSTIC}

\subsection{Caractéristiques du Site}

Bassin versant de $50 \mathrm{~km}^{2}$, plaine

Plusieurs communes

* amont rural : grande culture

* aval urbanisé récemment

Pluies de régime atlantique

\section{Instances \& Mission}

Origine des désordres, Apprécier leur récurrence

Appel en cause du lotisseur, Civil et administratif

\section{Investigations expertales}

Analyse météo de récurrence

Levers topo

Cartographie POS du B V

Schéma hydraulique contradictoire

\subsection{Caractéristiques du Site}

Bassin versant de $600 \mathrm{~km}^{2}$, pentu

$>10$ communes

* amont montagne : bois taillis

* aval vallées urbanisées

Pluies de régime méditerranéen intense

Exceptionnelles : $19 \mathrm{~h} \& 21 \mathrm{~h}$

\section{Instances \& Mission}

Origine des désordres : effet de vague, Apprécier leur récurrence

Mise en cause d'industrie du bois en amont et de retenue collinaire en haut BV

Civil et administratif

\section{Investigations expertales}

Analyse météo de fréquence

\& Imagerie radar

\& hydrologique : 1 station en aval BV

Etude hydraulique de BE

Constatations in situ

\subsection{Caractéristiques du Site}

Bassin versant de $40 \mathrm{~km}^{2}$, plateau

Plusieurs communes

* amont rural : taillis \& cultures

* aval urbanisé récemment

Pluies de régime atlantique

\section{du Sinistre}

Lotissement récent $>100$ lots

Submersions en plusieurs points

Plusieurs indemnisations CATNAT

Terrain rural urbanisé

sol saturé + Pluies continues + orage

\author{
Conclusions \\ 4 causes d'inondation du site \\ Creux topo, digues du ruisseau et de la rivière \\ + section de pont insuffisante, pluvial \\ Occurrence décennale ou moins \\ Sécurisation du site : digues, exhaure pluvial \\ Modèle hydrologique du BV
}

\section{du Sinistre}

Ville au confluent du BV + PER

Crue torrentielle : $5 \mathrm{~mm}^{3}$

Indemnisations CATNAT réalisée

Petite ville à urbanisation modernisée

Victimes, destruction de pont, habitations, Industries, > 100 véhicules,...

\section{Mission}

Origine des désordres : naturelle exceptionnelle ou liés aux aménagements

Apprécier leur récurrence versus la référence CATNAT

\section{Investigations expertales}

Analyse météo de stations

\& Imagerie radar

BV de référence

Etude hydraulique de BE

Constatations in situ

Cartographie POS du BV

\section{Conclusions}

Vague $=$ issue de la $2^{\circ}$ séquence pluviale Occurrence de crue 70 ans ou plus

Lac collinaire mis hors de cause : peu dégradé Scierie part marginale aux dégâts

Arrêt des procédures

Action permanente de prévention, à suivre

\section{du Sinistre}

ZAC industries \& commerces

Submersion généralisée + flux torrentiel

Indemnisation CATNAT lourde

industrie + commerce $=$ pertes d'exploitation

Crue de $2 \mathrm{~h}$ du matin

\section{Conclusions}

Occurrence estimée de la pluie 10 à 20 ans :

Aménagements en cause :

hydraulique (lit) + urbanisme industriel

Parc de grande surface à sécuriser

Action de prévention lourde à faire 


\subsection{Figures}

3.4.1 Agglomération inondée à l'exutoire du bassinversant

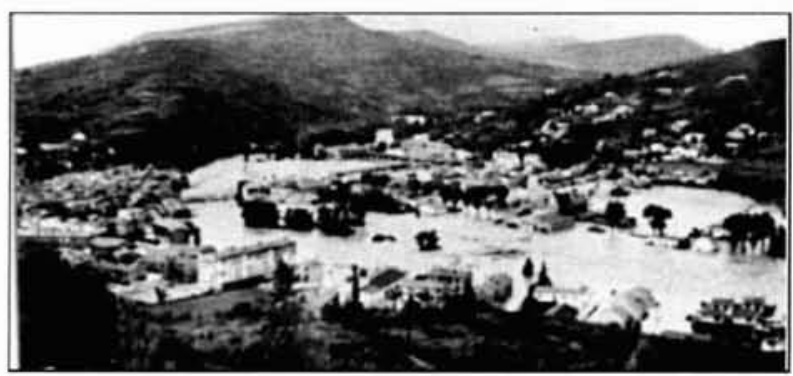

Types de dégradations produites par l'inondation torrentielle :

* Dans l'agglomération, à l'exutoire du bassin-versant.

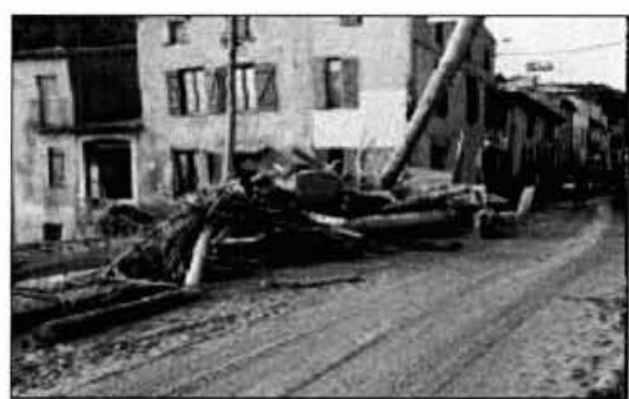

Billes de bois en amont du pont principal.

* Dans le haut bassin-versant rural.

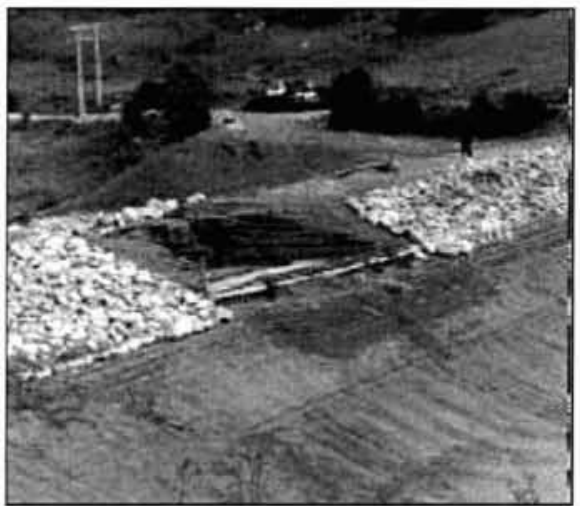

Déversoir de lac collinaire dégradé mais guide préservée.
3.4.2 Précipitations sur Imagerie radar avec tracés de $B V$ sinistrés

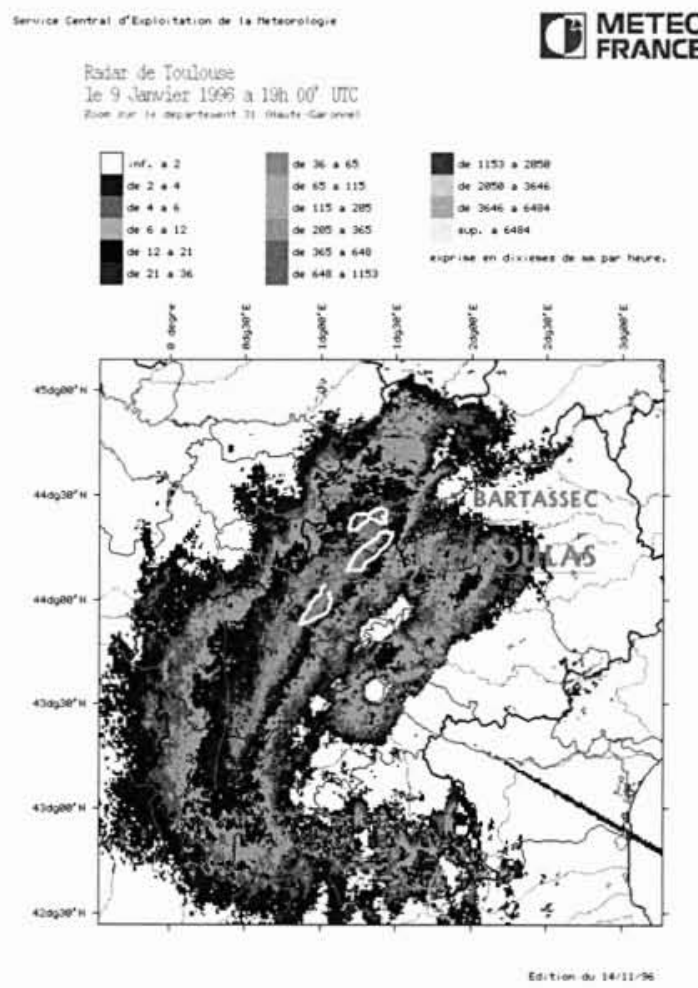




\subsubsection{Discontinuité entre POS voisins le long du cours d'eau}

Section nord : fortement urbanisé.

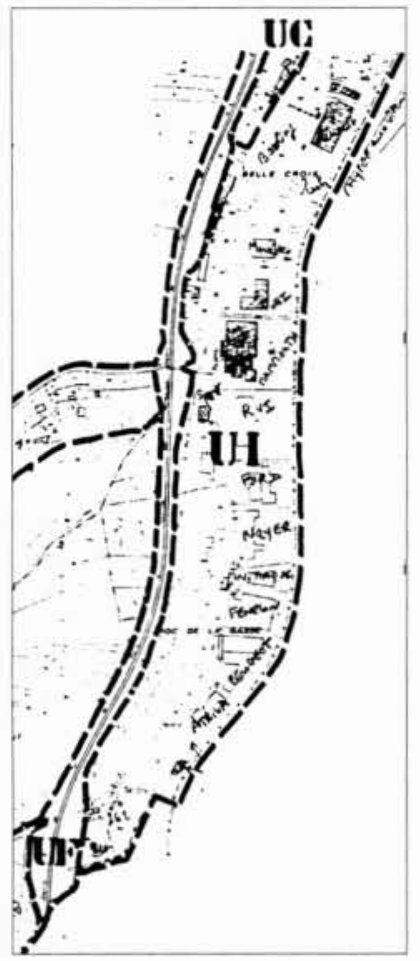

\section{IV $\square$ CONCLUSION}

Dans un contexte réglementaire plus contraignant les zones inondées sont devenues vulnérables par accumulation d'aménagements surtout le long des petits cours d'eau plus que des fleuves, causant des sinistres très coûteux.

Dans les sites devenus sensibles il convient d'engager à titre préventif un audit d'inondabilité au regard des critères d'assurance, et aussi pour se prémunir des litiges.

Sinon, lorsque surviennent les contentieux selon l'accroissement de demande sociale observée en matière de justice, ils relèvent maintenant de juridictions plus nombreuses, et fréquemment de plusieurs d'entre elles pour un même sinistre, ce qui contribue à allonger les délais de règlement des litiges et les difficultés des Maîtres d'Ouvrage.

Dans la logique de réhabilitation du site vulnérable, si le diagnostic d'inondabilité n'est qu'une étape, il apporte cependant un point de vue global. Mais à l'encontre de la tendance au laisser faire, il montre souvent que spéculer sur les probabilités n'est plus réaliste car un biais a été introduit
Section sud : rurale et inondable.

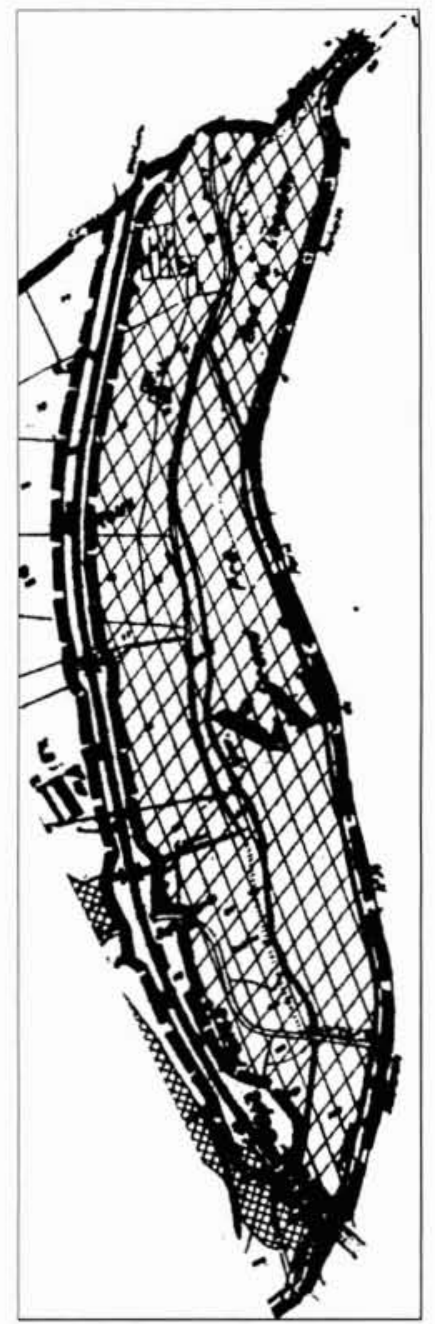

dans l'un des paramètres qui conditionne l'inondabilité, le plus souvent celui de l'aménagement.

Sur la base d'une segmentation des cas de sinistres, pour les petits cours d'eau devenus préoccupants, des mesures correctrices sont recommandées à la dimension hydro-géographique du site et techniquement et réglementairement cohérentes selon un échéancier de moyen terme.

La première étape pour cela reposera sur une modélisation à la mesure du bassin versant tant hydraulique que d'occupation du sol à travers un SIG. Cet outil permettra ensuite de choisir le meilleur scénario de développement pour un certain niveau de maîtrise des crues.

Vient ensuite une réglementation préventive, qui au-delà du PPR aboutira au POS Hydraulique du bassin versant pour toutes les Communes du BV concerné.

Alors que pour le court terme, il faudra accepter que la protection d'urgence et l'indemnisation jouent encore un rôle essentiel, il devient ainsi possible d'introduire progressivement et à moyen terme des infrastructures de protection contre les crues, dont le surcoût peut être planifié. 